\title{
Molecular and Serological Evaluation of Toxoplasma gondii Infection in Reared Turkeys in Fars Province, Iran
}

\author{
Bahador Sarkari ${ }^{1}$; Qasem Asgari ${ }^{2, *}$; Neda Bagherian ${ }^{2}$; Soheil Ashkani Esfahani ${ }^{3}$; Mohsen \\ Kalantari ${ }^{2}$; Iraj Mohammadpour ${ }^{2}$; Majid Ashrafmansori ${ }^{2}$; Maryam Amerinia ${ }^{1}$; Fatemeh \\ Sabet Sarvestani ${ }^{1}$ \\ ${ }^{1}$ Basic Sciences in Infectious Diseases Research Center, Shiraz University of Medical Sciences, Shiraz, IR Iran \\ ${ }_{3}^{2}$ Department of Parasitology and Mycology, School of Medicine, Shiraz University of Medical Sciences, Shiraz, IR Iran \\ ${ }^{3}$ Student Research Committee, Shiraz University of Medical Sciences, Shiraz, IR Iran \\ ${ }^{*}$ Corresponding author: Qasem Asgari, Department of Parasitology and Mycology, School of Medicine, Shiraz University of Medical Sciences, Shiraz, IR Iran. Tel/Fax: +98-7112305291, \\ E-mail: asgariq@sums.ac.ir
}

Received: May 8, 2013; Revised: June 29, 2013; Accepted: June 30, 2013

\begin{abstract}
Background: Toxoplasmosis is a parasitic disease caused by the protozoan Toxoplasma gondii. This parasite infects most of warm-blooded animals, including birds. Turkeys are one of these animals which might be infected by this parasite. Little is known about the prevalence of T. gondii in turkeys in Iran.

Objectives: The current study aimed to evaluate the rate of Toxoplasma infection in turkeys in Fars Province, Southern Iran.

Materials and Methods: Sera and tissues (brain, neck and tongue) of 54 turkeys were collected from Shiraz slaughterhouse in Fars province. Anti-Toxoplasma antibodies were assessed in the collected sera using modified agglutination test (MAT), while tissues were evaluated by polymerase chain reaction (PCR) and bioassay methods.

Results: T. gondii antibodies (MAT titer: $\geq 1: 40$ ) were found in $89.8 \%$ of turkeys. T. gondii DNA was detected in $61.6 \%$ of turkey tissues and brain had the highest rate of infection. Brain tissues from each animal were bioassayed and Toxoplasma tissue cysts were found in $11.5 \%$ and Toxoplasma DNA in $62 \%$ of inoculated mice.

Conclusions: Results of this study validated a relatively high level of Toxoplasma infection in reared turkeys and turkey meat might be considered as an infection sources for human.
\end{abstract}

Keywords:Toxoplasma; Polymerase Chain Reaction; Biological Assay; Turkeys

\section{Background}

Toxoplasma gondii is a protozoan which can infect humans and warm-blooded domestic and wild animals including birds. Humans are commonly infected with $T$. gondii by consumption of oocysts present in soil or water, polluted with cat feces, or ingestion of tissue cysts in underdone meat (1-3). Nevertheless, transmission of $T$. gondii is more likely through ingestion of infected tissues than ingestion of oocysts (1).

The infection is particularly important in women when they acquire the infection for the first time during their pregnancy, where an intrauterine transmission of the parasite may occur. Congenital toxoplasmosis affects 1-10 per 10000 infants in Europe. Its incidence and severity vary depending on the time of infection in mothers. Transmission rate increases with gestational age, whereas the severity of infection reduces by the time of infection. Food animals including pigs, sheep, goats, and reared birds, such as chickens and turkeys, can be infected by Toxoplasma and these animals can transmit the infection to humans through their meats. Birds are important intermediate hosts of Toxoplasma, since they serve as a source of infection for humans as well as an important reservoir for cats (4). Human toxoplasmosis is a common protozoan infection in Iran (5). Seroprevalence rate of toxoplasmosis in general population of Iran has been reported 51.8\%(5). This rate varies in different parts of the country. Seroprevalence rates of 55\% and $29 \%$ have been reported for toxoplasmosis in northern and southern parts of Iran, respectively. In Fars province, southern Iran, seroprevalence rate of toxoplasmosis in sheep and goats have been reported $26.5 \%$ and $14.02 \%$, respectively (6). Toxoplasma infection in human occurs commonly through consumption of undercooked or raw meat. Infected lamb meat is considered as one of the main sources of Toxoplasma infection in human (7). In a recent study, Toxoplasma infection in edible tissues of sheep and goats in Fars province was evaluated by molecular methods. The study revealed

Implication for health policy/practice/research/medical education:

Meat of breeding turkeys might be infected with Toxoplasma and consumption of these meats might be a source of Toxoplasma infection for humans.

Copyright @ 2014,Ahvaz Jundishapur University of Medical Sciences; Published by Kowsar Corp. This is an open-access article distributed under the terms of the Creative Commons Attribution License, which permits unrestricted use, distribution, and reproduction in any medium, provided the original work is properly cited. 
that five of 22 goats (22.7\%) and 21 of 56 sheep (37.5\%) were infected by Toxoplasma (8). Cats are the final hosts for Toxoplasma and rate of infection in these reservoir hosts has been relatively high in Iran (9). In the recent years, breeding of turkeys has been quite common in Iran, including Fars province. Meat of these birds might be infected by Toxoplasma and transfer the infection to humans. Prevalence of Toxoplasma infection in turkeys varies in different parts of the world, ranging from $\leq 1 \%$ to $80 \%(10-12)$.

\section{Objectives}

The current study evaluated seroprevalence of T. gondii in reared turkeys in Fars province, southern Iran. Moreover, the study aimed to assess the rate of Toxoplasma infection using molecular methods in animal tissues. The study was justified by the lack of information about prevalence of T. gondii in turkeys in Iran.

\section{Materials and Methods}

\subsection{Study Area and Sample Collection}

The current study was conducted in 2012 in Fars province, southwest of Iran Farms for rearing free-range turkeys have been growing during the last decades in this region. Sera and tissue samples (brain, neck muscle and tongue) were collected from 54 turkeys from slaughterhouse in Shiraz from April to September 2012. Collected sera were evaluated for anti-Toxoplasma antibodies by modified agglutination test (MAT). Tissue samples were assessed for Toxoplasma infection by PCR and bioassay methods.

\subsection{Modified Agglutination Test}

MAT was performed using formalin-fixed whole $\mathrm{RH}$ tachyzoites, as originally explained by Desmonts and Remington (13). Briefly, the test was performed, using formalin-fixed Toxoplasma tachyzoites. Turkeys and mice serum samples were diluted with phosphate buffered saline (PBS, $\mathrm{pH}=7.2$ ), and evaluated for anti-Toxoplasma IgG antibodies. The antigens were diluted with antigen diluting buffer, containing bovine serum albumin (BSA), 2-mercaptoethanol and Evans blue dye solution. Positive and negative sera, as controls, were incorporated in each plate with same dilutions as the test sera. Sera with MAT titers of 1:40 or higher were considered as positive. A blue pellet at the base of the U-shape-well microtiter plates (Nunc) were considered as negative, while agglutination of the formalin-fixed parasite in a mat, covering about half of the well base, was recorded as positive.

\subsection{Detection of T. gondii in Turkey Tissues by PCR}

DNA was extracted from $1 \mathrm{~g}$ of the homogenized tissues. Proteinase $\mathrm{K}$ and lysis buffer ( $1 \mathrm{mM}$ of EDTA, $\mathrm{pH}=$ 8.0; $50 \mathrm{~mL}$ of Tris- $\mathrm{HCl}, \mathrm{pH}=7.6 ; 1 \%$ of Tween 20 ) were added to the samples and incubated at $37^{\circ} \mathrm{C}$ for 24 hours. Phenol/chloroform/isoamyl alcohol was used to extract the lysate, and the DNA was precipitated using absolute ethanol (8). Nested primer sets (Bioneer, Korea) were used to amplify the fragments of the B1 gene, as previously reported (8). Briefly, outer primers ( $5^{\prime}$-CCG TTGGTT CCG CCT CCT TC- $3^{\prime}$ and $5^{\prime}$-GCA AAA CAG CGG CAGCGT CT-3`), producing an amplified product of $432 \mathrm{bp}$ and inner primers, (5 -CCG CCT CCT TCG TCCGTC GT-3` and 5 '-GTG GGG GCG GAC CTC TCT TG-3`'), producing an amplified product of $213 \mathrm{bp}$ were used. Details of the PCR program are given in Tables 1 and 2.

\subsection{Bioassay in Mice}

Brain tissues of turkeys were inoculated intraperitoneally into mice. All the mice were monitored daily over two months. After that, blood samples were taken from the mice for serological assays and their brains were removed for cyst detection and DNA extraction. Impression smears were prepared from livers, spleens and brains of mice for detection of tachyzoites or tissue cysts. Tissues of inoculated mice were examined for Toxoplasma DNA by PCR.

\subsection{Statistical Analysis}

All statistics were performed using SPSS version 16 statistical package. Chi-square test was used to determine the influence of examined factors on seropositivity to Toxoplasma. P value less than 0.05 was considered statically significant. Degree of agreement was quantified by kappa statistic.

\begin{tabular}{|c|c|c|c|c|c|}
\hline Program Toxo 1 & Segment & Definition & Temperature, ${ }^{\circ} \mathrm{C}$ & Time, min & No. of Cycles \\
\hline Program 1 & 1 & Heat start (amplification) & 94 & 2 & 1 \\
\hline \multicolumn{6}{|l|}{ Program 2} \\
\hline & 1 & Denaturation & 94 & 1 & 30 \\
\hline & 2 & Annealing & 57 & 2 & 30 \\
\hline & 3 & Extension & 72 & 3 & 30 \\
\hline Program 3 & 1 & Final & 72 & 10 & 1 \\
\hline Program 4 & 1 & - & 4 & 99/99 & - \\
\hline
\end{tabular}


Sarkari B et al.

\begin{tabular}{|c|c|c|c|c|c|}
\hline Program Toxo 1 & Segment & Definition & Temperature, ${ }^{\circ} \mathrm{C}$ & Time, min & No. of Cycles \\
\hline Program 1 & 1 & Heat start (amplification) & 94 & 2 & 1 \\
\hline \multicolumn{6}{|l|}{ Program 2} \\
\hline & 1 & Denaturation & 94 & 1 & 35 \\
\hline & 2 & Annealing & 58 & 1 & 35 \\
\hline & 3 & Extension & 72 & 1 & 35 \\
\hline Program 3 & 1 & Final & 72 & 10 & 1 \\
\hline Program 4 & 1 & - & 4 & 99/99 & - \\
\hline
\end{tabular}

Table 3. Molecular and Serological Rate of Toxoplasma Infection in Reared Turkeys in Southern Iran ${ }^{a}$

\begin{tabular}{lcccccccc}
\hline PCR & \multicolumn{2}{c}{ Neck } & \multicolumn{2}{c}{ Brain } & \multicolumn{2}{c}{ Tongue } & Total \\
\hline MAT & Positive & Negative & Positive & Negative & Positive & Negative & Positive & Negative \\
\hline Positive & 9 & 24 & 11 & 22 & 8 & 25 & 27 \\
Negative & 1 & 6 & 2 & 5 & 2 & 5 & 3 & 4 \\
\hline
\end{tabular}

a Abbreviations: MAT, modified agglutination test; PCR, polymerase chain reaction.

\section{Results}

T. gondii antibodies (MAT titer: $\geq 1: 40$ ) were found in $89.8 \%$ of turkeys. PCR detected 351- and 546-bp bands, corresponding to Toxoplasma infection, in tissues of 33 of 54 (61.6\%) turkeys. Brain was the most infected tissue (33.3\%), followed by tongue (31.5\%) and neck (29.6\%) tissues (Table 3). Brain tissues from each animal were bioassayed and Toxoplasma tissue cysts were found in $11.5 \%$ and Toxoplasma DNA in $62 \%$ of inoculated mice. Coinfection of brain and neck was noticed in eight, brain and tongue in 12, and neck and tongue in eight turkeys. MAT was positive in 16 PCR-negative animals and was negative in three PCR-positive cases. The agreement between PCR and MAT was 62\%. Statistical analysis of MAT and PCR findings showed that there were no significant correlation between seropositivity and PCR positivity of brain, neck and tongue tissues. Results of bioassay in mice revealed tissue cysts in brain of $11.5 \%$ of the inoculated mice, while PCR detected Toxoplasma infection in brain of 31 of 50 (62\%) inoculated mice.

\section{Discussion}

Nearly one third of the world population is infected by Toxoplasma and consumption of underdone meat is considered as the main causes of infection (2). The disease is mainly asymptomatic in immune-competent individuals and clinical signs may be present in few of the infected people (1). Toxoplasma infection is common in various animals including sheep, pigs, goat, and poultry, which are used for food. Infection in other animals such as cattle, horses, and buffaloes is less prevalent. Domestic turkeys are usually resistant to clinical toxoplasmosis (4). Results of experimental infections demonstrated that even one or two-week-old turkeys inoculated with millions of tachyzoites of RH strains became infected, but did not de- velop clinical signs, while all of experimentally infected turkeys harbored T. gondii in their tissues (14). Similar results were obtained by feeding $T$. gondii oocysts to turkeys, where turkeys were fed with oocysts and viable $T$. gondii were recovered from tissues of all turkeys by bioassays in mice and all the turkeys developed antibodies to $T$. gondii, detectable by MAT (15).

In this study, we used MAT for detection of anti-T. gondii antibodies in the reared turkeys or mice. MAT is based on detection of IgG antibodies, produced by the host against Toxoplasma, and uses formalin-fixed Toxoplasma tachyzoites. MAT is an appropriate test for seroprevalence studies of toxoplasmosis in animals since it does not need speciesspecific conjugates usually used in IFA or ELISA methods. Sensitivity and specificity of MAT has been reported $82.9 \%$ and $90.29 \%$, respectively, in comparison with other commercially available serological assays for detection of Toxoplasma IgG antibodies in animals. MAT is a method extensively used for seroprevalence studies in the recent years (16-21).

Findings of this study revealed that more than $80 \%$ of reared turkeys in southern Iran were infected with Toxoplasma. Comparing the rate of Toxoplasma infection in this study with currently available reports from different geographical areas, higher prevalence of Toxoplasma in turkeys can be seen in this area $(10,11,22,23)$. In Germany, seroprevalence of T. gondii in 1913 turkeys from 14 turkey farms from different areas of the country was $20.2 \%$ (ranging from zero to $77.1 \%$ ) (10). An antibody prevalence of $10 \%$ has been reported in turkeys of the southeastern United States (22). In another study, anti-Toxoplasma antibodies were detected in 12 of 17 sera from turkeys of Alabama (12). In Egypt, T. gondii seroprevalence was examined in turkeys, showing prevalence rate of $29.4 \%$ (11).

High level of seropositivity in our study, compared with 
other studies, might be linked to high level of T. gondii infection in animal and also human in our region. In a recent study, conducted by the author of the current study, seroprevalence of toxoplasmosis in farm animals was evaluated. Sera from farm animals including cows, dogs, horses, sheep and goats, were serologically assessed for anti-Toxoplasma antibodies. Result showed that 121 of 346 (34.9\%) animals were infected by Toxoplasma. Cattle had the highest rate of infection, followed by dogs, horses, sheep and goat (24).

Raeghi et al. evaluated seroprevalence of toxoplasmosis in Urmia, capital of West Azerbaijan province, Iran, and found that $21.1 \%$ of sheep, $1.6 \%$ of cattle, and $11.5 \%$ of horses were seropositive for $T$. gondii. They also reported that sheep were 15 times more likely to be seropositive for Toxoplasma, compared with cattle (25). Tissue cysts of T. gondii may last in food animals for years. Almost all edible portions of an animal can comprise viable $T$. gondii. Rate of T. gondii tissue cysts in meat of food animals is usually low. In 100 grams of meat, as few as one tissue cyst may be present; thus, it is difficult to detect this low level of $T$. gondii infection in tissues of animals, unless a reliable molecular approach is used.

T. gondii can be isolated from animals by inoculation of laboratory animals with animal tissues. Microscopical detection of Toxoplasma tissue cyst in tissues of animals is not a reliable method. Moreover, histochemical or immunohistochemical staining of parasites are not sensitive methods for detection of T. gondii in animal tissues (26). In several studies, detection of T. gondii DNA in meat of animals or human tissues by molecular approaches has been described $(8,24,27,28)$. However, efficacy of these methods has not appropriately been defined. Improved methods such as real-time PCR and fluorogenic probe can detect DNA of as few as four bradyzoites of $T$. gondii in tissues or other samples of humans or animals. T. gondii isolates of human and animal sources are clustered into three clonal types, I, II and III (29). Features of these lineages are different in a variety of aspects including their virulence in laboratory animals. While infection with type I is lethal for outbred mice, infections with types II and III are usually less virulent in these hosts. Type II strains are the main common types of Toxoplasma in North America and Europe.

Prevalence and genotypes of Toxoplasma in avian hosts (free-range chickens, sparrows, pigeons and starlings) in the southwest of Iran (Khuzestan province) has been evaluated by Khademvatan et al. (19). The results demonstrated that $16.5 \%$ of samples of avian tissues were infected with T. gondii. Molecular typing and DNA sequencing found types II and III T. gondii as the predominant lineages of the parasites. Moreover, a higher prevalence rate of type III, compared with type II, was reported in their study (19). Results of the current study were in accordance with those showing a high prevalence of Toxoplasma in this region in animals and humans.

Brain was the most infected tissue in our study, followed by neck muscle and tongue. Although seropositivity of turkeys is not directly linked to infection of tissues, however, results of bioassays in mice demonstrated that tissues of seropositive turkeys can induce infection in mice. It should be highlighted that seropositivity of turkeys to Toxoplasma does not necessarily mean the infectivity for consumers, except for birds with positive bioassay results. Supporting this point, some seropositive turkeys were PCR-negative. Considering the high rate of T. gondii infection in turkeys in the region, undercooked eatable tissues of these birds must be considered as sources of Toxoplasma infection for humans in the region. Proper cooking of turkey meat can be recommended for preventing the transmission of Toxoplasma to human. Moreover, not feeding raw or undercooked meat to cats and keeping cats outside the turkey breeding farms will reduce the risks of infection from cats to turkeys.

\section{Authors' Contributions}

Bahador Sarkari and Qasem Asgari participated in the study design, conducted the experiments, and drafted the manuscript. The rest of authors collected the samples and conducted the experiments.

\section{Financial Disclosure}

The authors had no financial interests related to the materials in this manuscript.

\section{Funding/Support}

This study was financially supported by the office of vice-chancellor for research of Shiraz University of Medical Sciences (Grant no. 89-01-43-2007).

\section{References}

1. Dubey JP, Jones JL. Toxoplasma gondii infection in humans and animals in the United States. Int J Parasitol. 2008;38(11):1257-78.

2. Elmore SA, Jones JL, Conrad PA, Patton S, Lindsay DS, Dubey JP. Toxoplasma gondii: epidemiology, feline clinical aspects, and prevention. Trends Parasitol. 2010;26(4):190-6.

3. Cenci-Goga BT, Rossitto PV, Sechi P, McCrindle CM, Cullor JS. Toxoplasma in animals, food, and humans: an old parasite of new concern. Foodborne Pathog Dis. 2011;8(7):751-62.

4. Dubey JP. A review of toxoplasmosis in wild birds. Vet Parasitol. 2002;106(2):121-53.

5. Assmar M, Amirkhani A, Piazak N, Hovanesian A, Kooloobandi A Etessami R. [Toxoplasmosis in Iran. Results of a seroepidemiological study]. Bull Soc Pathol Exot. 1997;90(1):19-21.

6. Asgari Q, Moazzeni M, Akrami Mohajeri F, Kalantari M, Zarifi M Ghalebi SR, et al. Seroprevalence of Toxoplasma gondii among caprines in Fars province, Southern Iran.JVet Parasitol.2007;21(2):153-5.

7. Dubey JP. Toxoplasmosis in sheep--the last 20 years. Vet Parasitol. 2009;163(1-2):1-14.

8. Asgari Q, Sarnevesht J, Kalantari M, Sadat SJ, Motazedian MH Sarkari B. Molecular survey of Toxoplasma infection in sheep and goat from Fars province, Southern Iran. Trop Anim Health Prod. 2011;43(2):389-92.

9. Hooshyar H, Rostamkhani P, Talari S, Arbabi M. Toxoplasma gondii infection in stray cats. Iranian J Parasitol. 2007;2:18-22.

10. Koethe M, Pott S, Ludewig M, Bangoura B, Zoller B, Daugschies A, et al. Prevalence of specific IgG-antibodies against Toxoplasma gondii in domestic turkeys determined by kinetic ELISA based on 


\section{Sarkari B et al.}

recombinant GRA7 and GRA8. Vet Parasitol. 2011;180(3-4):179-90.

11. Harfoush M, Tahoon Ael N. Seroprevalence of Toxoplasma gondii antibodies in domestic ducks, free-range chickens, turkeys and rabbits in Kafr El-Sheikh Governorate Egypt. J Egypt Soc Parasitol. 2010;40(2):295-302.

12. Lindsay DS, Smith PC, Blagburn BL. Prevalence and isolation of Toxoplasma gondii from wild turkeys in Alabama. J Helminthol Soc Wash. 1994;61(1):115-7.

13. Desmonts G, Remington JS. Direct agglutination test for diagnosis of Toxoplasma infection: method for increasing sensitivity and specificity. JClin Microbiol.1980;11(6):562-8.

14. Drobeck HP, Manwell RD, Berstein E, Dillon RD. Further studies of toxoplasmosis in birds. Am JHyg. 1953;58(3):329-39.

15. Dubey JP, Camargo ME, Ruff MD, Wilkins GC, Shen SK, Kwok OC, et al. Experimental toxoplasmosis in turkeys. J Parasitol. 1993;79(6):949-52.

16. Alvarado-Esquivel C, Estrada-Malacon MA, Reyes-Hernandez SO, Perez-Ramirez JA, Trujillo-Lopez JI, Villena I, et al. Seroprevalence of Toxoplasma gondii in domestic sheep in Oaxaca State, Mexico. JParasitol. 2013;99(1):151-2.

17. Xu P, Song X, Wang W, Wang F, Cao L, Liu Q. Seroprevalence of Toxoplasma gondii infection in chickens in Jinzhou, northeastern China. J Parasitol. 2012;98(6):1300-1.

18. Garcia-Bocanegra I, Cabezon O, Arenas-Montes A, Carbonero A, Dubey JP, Perea A, et al. Seroprevalence of Toxoplasma gondii in equids from Southern Spain. Parasitol Int. 2012;61(3):421-4.

19. Khademvatan S, Saki J, Yousefi E, Abdizadeh R. Detection and genotyping of Toxoplasma gondii strains isolated from birds in the southwest of Iran. Br Poult Sci. 2013;54(1):76-80.

20. Hajialilo E, Ziaali N, Harandi MF, Saraei M, Hajialilo M. Prevalence of anti-Toxoplasma gondii antibodies in sport horses from Qazvin, Iran. Trop Anim Health Prod. 2010;42(7):1321-2.
21. Hamidinejat H, Ghorbanpour M, Nabavi L, Haji Hajikolaie MR, Razi Jalali MH. Occurrence of anti-Toxoplasma gondii antibodies in female cattle in south-west of Iran. Trop Anim Health Prod. 2010;42(5):899-903.

22. Quist CF, Dubey JP, Luttrell MP, Davidson WR. Toxoplasmosis in wild turkeys: a case report and serologic survey. J Wildl Dis. 1995;31(2):255-8.

23. El-Massry A, Mahdy OA, El-Ghaysh A, Dubey JP. Prevalence of Toxoplasma gondii antibodies in sera of turkeys, chickens, and ducks from Egypt. J Parasitol. 2000;86(3):627-8.

24. Asgari Q, Sarkari B, Amerinia M, Panahi S, Mohammadpour I, Sadeghi Sarvestani A. Toxoplasma Infection in Farm Animals: A Seroepidemiological Survey in Fars Province, South of Iran. Jundishapur J Microbiol. 2013;6(3):269-72.

25. Raeghi S, Akaberi A, Sedeghi S. Seroprevalence of Toxoplasma gondii in Sheep, Cattle and Horses in Urmia North-West of Iran. Iran JParasitol. 2011;6(4):90-4.

26. Sarkari B, Asgari Q, Mirzaei S. Evaluation of immunohistochemistry and PCR in diagnosis of toxoplasma infection in tissues of human aborted fetuses. Zahedan J Res Med Sci. 2013;15(12):42.

27. Rahdar M, Samarbaf-Zadeh A, Arab L. Evaluating the Prevalence of Toxoplasma gondii in Meat and Meat Products in Ahvaz by PCR Method. Jundishapur J Microbiol. 2012;5(4):570-3.

28. Asgari Q, Fekri M, Monabati A, Kalantary M, Mohammadpour I, Motazedian MH, et al. Molecular Genotyping of Toxoplasma gondii in Human Spontaneous Aborted Fetuses in Shiraz, Southern Iran. Iran J Public Health. 2013;42(6):620-5.

29. Sibley LD, Khan A, Ajioka JW, Rosenthal BM. Genetic diversity of Toxoplasma gondii in animals and humans. Philos Trans $R$ Soc Lond B Biol Sci. 2009;364(1530):2749-61. 